\title{
Tailored Displays to Compensate for Visual Aberrations
}

\author{
Vitor F. Pamplona ${ }^{1}$ \\ Manuel M. Oliveira ${ }^{1}$ \\ ${ }^{1}$ Instituto de Informática - UFRGS \\ ${ }^{2}$ Purdue University \\ Daniel G. Aliaga ${ }^{2} \quad$ Ramesh Raskar $^{3}$
${ }^{3}$ Camera Culture Group - MIT Media Lab
}

\begin{abstract}
We introduce tailored displays that enhance visual acuity by decomposing virtual objects and placing the resulting anisotropic pieces into the subject's focal range. The goal is to free the viewer from needing wearable optical corrections when looking at displays. Our tailoring process uses aberration and scattering maps to account for refractive errors and cataracts. It splits an object's light field into multiple instances that are each in-focus for a given eye subaperture. Their integration onto the retina leads to a quality improvement of perceived images when observing the display with naked eyes. The use of multiple depths to render each point of focus on the retina creates multi-focus, multi-depth displays. User evaluations and validation with modified camera optics are performed. We propose tailored displays for daily tasks where using eyeglasses are unfeasible or inconvenient (e.g., on head-mounted displays, ereaders, as well as for games); when a multi-focus function is required but undoable (e.g., driving for farsighted individuals, checking a portable device while doing physical activities); or for correcting the visual distortions produced by high-order aberrations that eyeglasses are not able to.
\end{abstract}

Keywords: tailored displays, optics, light-field displays

Links: $\odot$ DL PDF WEB

\section{Introduction}

Current high-resolution displays switch the limits of visual performance from the usual pixel density to the users' own visual acuity. Uncorrected eye aberrations decrease one's visual capabilities, creating sub-optimal, uncontrolled and heterogeneous user experiences. Further improvements on display resolution will only be appreciated by those with an over-standard eyesight. Acuity enhancement options range from simple eyeglasses to laser eye surgery. Wearable optical corrections are, however, inconvenient in several daily activities, such as during the practice of sports. In this paper, we explore novel image-enhancement techniques for visual performance based on light-field displays. Our approach creates hologram-style imagery that adjust themselves to the subject's eye conditions. This to some extent is similar to a still-under-research adaptive-optics-based contact lenses [Liang et al. 1997], but applied to the display instead of the eye. Our tailored displays account for refractive errors (e.g., nearsightedness, farsightedness, age-related visual degradation, astigmatism, and higher-order aberrations) and avoids scattering media on light paths, such as cataracts (Figure 1). To the best of our knowledge, this is the first user validated step towards a corrective-eyewear-free display.

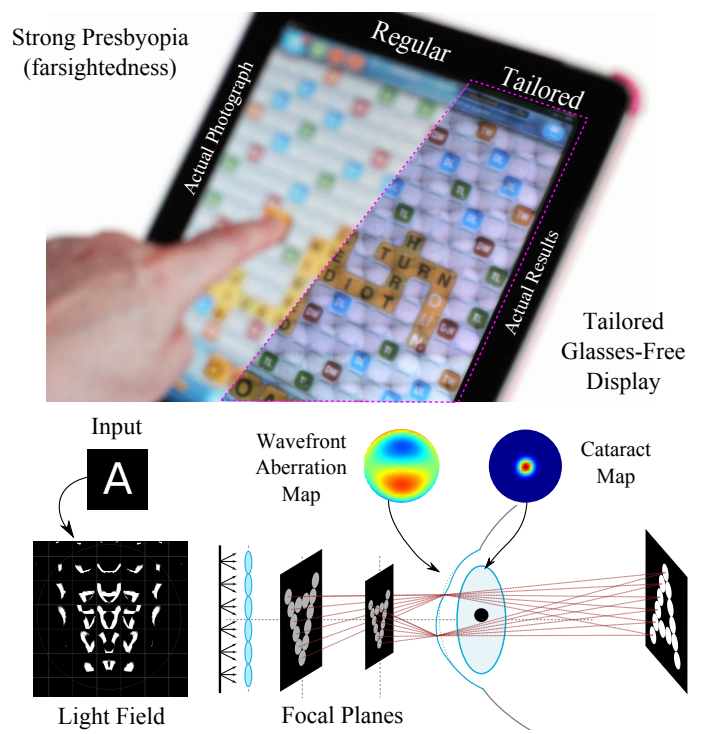

Figure 1: Can we create a display that adapts itself to improve one's eyesight? Top figure compares the view of a 2.5-diopter farsighted individual in regular and tailored displays. In the bottom, a 5-diopter nearsighted subject with nuclear cataracts and optical coma of 2 diopters observes an improved view of the letter $\mathbf{A}$. We use currently available inexpensive technologies to warp light fields to compensate for refractive errors and scattering sites in the eye.

Refractive errors and cataracts can be measured using modified parallax barriers in close range [Pamplona et al. 2010; Pamplona et al. 2011], or via ophthalmic tools that range from high-end ShackHartmann wavefront sensing systems [Liang et al. 1994; Donnelly et al. 2004] to low-cost Snellen-chart-based visual acuity tests. Our tailored light fields correct for visual aberrations using a lenslet array on top of a high-resolution display or a small stack of LCDs. We use aberration and scattering maps to pre-warp a light field, which virtually places the distorted anisotropic images into in-focus subaperture-dependent depths. The result is an enhanced projection onto the retina, with significant decrease of out-of-focus blur.

\subsection{Contributions}

We propose tailored displays to compensate for spatially-varying optical distortions of the human eye. Our contributions include:

- Multi-depth displays that compensate for aberrated vision and enhance uncorrected visual acuity. It supports time-varying optical corrections with no moving parts and uses off-theshelf components. We exploit current inexpensive technology to provide resolution around the standard retinal resolution;

- A real-time rendering procedure for 3D displays which distributes virtual objects and their light fields into many focal depths according to the wavefront aberrations of the eye's aperture. Virtual objects placed inside one's accommodation range are tailored to compensate for cataract and refractive effects. An extension to support multi-focus is also presented;

- Evaluation with human subjects and cameras under myopia, presbyopia, hyperopia, astigmatism, keratoconus (high-order aberration) and cataracts with binary and color pictures. 
To our knowledge, ours is the first display technology capable of adjusting itself to the observer's visual limitations, taking into account low and high-order aberrations, with no moving parts.

\subsection{Related Work}

Eyeglasses with simple, bifocal, and multi-focal lenses, pinhole glasses, contact lenses, LASIK and cataract surgeries, fiber optic tapers, adaptive spectacles [Sugiura and Morita 1993; Douali and Silver 2004], and adaptive optics [Thibos et al. 1999; Liang et al. 1997] can be used to enhance visual acuity. However, they require wearing prosthesis or making incisions in the eye.

Eye Modeling and Measuring Techniques Barsky [2004] proposed vision realistic rendering by using wavefront data to render images that simulate the subject's vision. Deering [2005] used a model of cones in the retina to simulate the perception of digital images. Camp et al. [1990] developed a rendering technique that accounts for eye aberrations based on corneal topography. Although these works have pushed the scientific frontier, none of them proposes a solution to enhance visual acuity, and some do not account for individual variability but for an average response. Pamplona et al. [2010; 2011] pointed out a duality between Shack-Hartmann and light-field techniques and showed how computer graphics can be used to measure and model some eye aberrations. Tailored displays tackle the inverse problem of measuring techniques, correcting for the measured aberrations and providing an improved acuity.

Vision Enhancement and 3D Displays Our system uses glassesfree 3D display hardware (parallax barriers [Ives 1903; Isono et al. 1993] and lenticular-based displays [Lippmann 1908]) to overcome one's limitation to focus. Lanman et al. [2010] uses retinal integration to improve cues for convergence, while Wetzstein et al. [2011] recently discussed the benefits of a mask/LCD stack with more than two layers. Instead of dealing with convergence cues, we use 3D displays to compensate for aberrations on the eye. Multi-focus displays, such as Akeley et al. [2004] and Hoffman et al. [2005], can enhance visual acuity by projecting images on the subject's range of accommodation. Goldring et al. [2006] has adapted a display with lenses to focus the image on the center of the eye lens improving readability. In the same way, Liu and Hua [2009], and Rolland et al. [2000] have introduced dynamic lenses that change the plane of focus and a stack of planes that are selectively illuminated to create volumetric head-mounted displays. These techniques, however, require moving parts and do not account for non-symmetric aberrations, such as astigmatism, higher-order aberrations, and cataracts. Thibos et al. [1999] use wearable liquid-crystal spatial modulators to reshape the wavefront of light. Their work is sound but limited to 1.5 diopters with current technology. Alonso et al. [2007] use Fourier optics and the Wiener filter to invert the eye's point spread function and display a deconvolved image on a standard monitor, achieving 3\% improvement on visual recognition tests. Huang and Barsky [2011] perform simulations of an "inverse blurring" operation to distort an image that would then be blurred by the out-offocus eye (no cataracts and light fields involved). Prototyping their conceptual multi-layer display does not seem to be a simple task. It requires, among other things, the use of multiple high-dynamicrange transparent displays, which its authors acknowledge are not realizable with current technology. We add to the growing body of glasses-free 3D display research by exploring a new degree of freedom: the user's eye aberrations. Super-resolution screens [Didyk et al. 2010] and superimposed projectors [Jaynes and Ramakrishnan 2003; Damera-Venkata and Chang 2009] used in light-field systems can be tailored to achieve visual acuity beyond standard eyes.

Light-Field Cameras and Refocusing Our work is the dual of light-field camera correction. The optical basics of our technique is

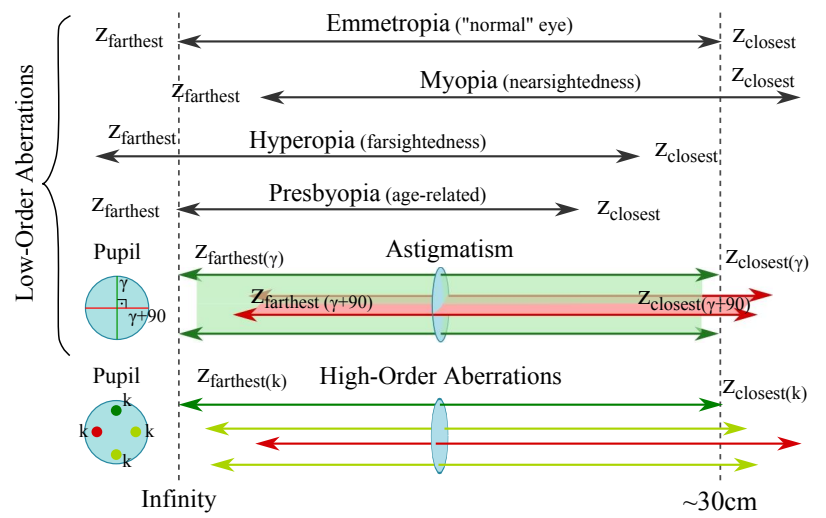

Figure 2: Focal/Accommodation range of distinct vision throughout aberrations. Myopia shifts the range closer, causing bad far sight. Hyperopia shifts the focal range farther, blurring near sight. Presbyopia reduces the accommodation power due to aging. Astigmatism has two principal focal lengths in perpendicular meridians due a toric curvature of the cornea or lens. Higher-order aberrations such as coma comprise of several focal lengths. Astigmatism and higher-order aberrations cannot project sharp images on the retina, blurring objects inside the subject's focal range.

described in [ $\mathrm{Ng}$ and Hanrahan 2006; Levoy et al. 2009]. Isaksen et al. [2000] introduce photographic effects in light-field rendering. Synthetic aperture concepts and camera calibration methods are discussed in [Vaish et al. 2004; Levoy et al. 2004]. Ng [2005] and $\mathrm{Ng}$ et al. [2005] demonstrate dynamic refocusing using light fields. Dai et al. [2009] and Jeong et al. [2005] propagate wavefront aberrations based on Zernike polynomials. In contrast, we center on the display and add support for refractive errors and cataracts.

\section{Acuity, Refractive Errors and Cataracts}

Cataracts and refractive errors are the main causes of loss of visual acuity worldwide. Approximately two billion people need eyeglasses [WHO 2005] and the prevalence of both conditions are expected to grow with the increasing longevity [EDPRG 2004] and heavy "near work", such as the use of electronic gadgets [Schaeffel 2006]. For instance, in urban areas of east Asia, $80-90 \%$ of children completing high school are now myopic [Morgan et al. 2012]. The term emmetropia (absence of refractive errors and cataracts i.e., normal vision) refers to the standard visual acuity of 1 arc minute (the smallest discernible feature of a typical eye). Theoretical limits on the human foveal vision are, however, found to be between 0.6 and 0.25 arc minutes [Schwiegerling 2000]. By increasing the optical quality of the image reaching the retina, even emmetropes could potentially double their visual performance.

Refractive errors misfocus objects onto the retina. Refractive powers/errors are expressed in diopters (D), the reciprocal of lens' focal length given in meters $\left(D=1 / f_{m}\right)$. Figure 2 illustrates the focal ranges for individuals with myopia, hyperopia, loss of accommodation power (presbyopia), radial asymmetry (astigmatism), and higher-order aberrations. Low-order aberrations are described in terms of spherical powers $(S)$ for symmetric errors (i.e., myopia, hyperopia, presbyopia); and cylindrical powers $(C)$ with an axis $(\gamma)$ for astigmatism. Higher-order aberrations are described using a spatial distribution of focal lengths (called wavefront map). Low-order aberrations can be converted to wavefront maps by fitting Zernike polynomials. Alternatively, the refractive power (in diopters) associated with a given eye meridian $\theta$ can be estimated as $P(\theta)=S+C \sin ^{2}(\gamma-\theta)$. Measuring techniques for refractive conditions include: (i) Snellen charts to check for visual acu- 


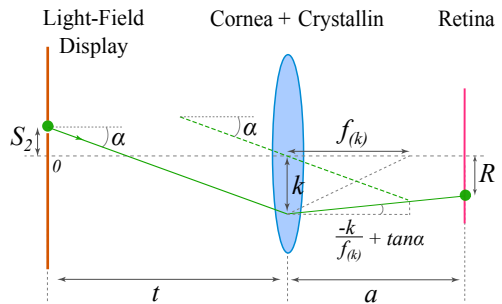

(a) Light-field Display

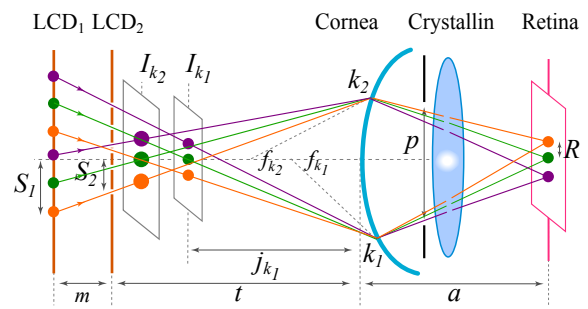

(b) Dual Stack of LCDs Display

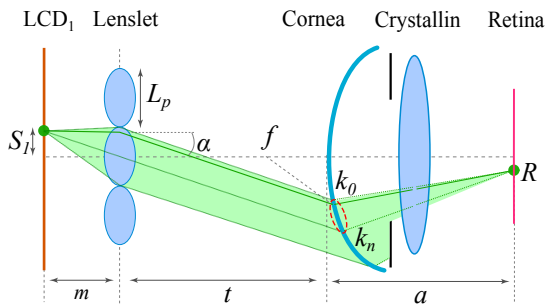

(c) Lenticular Array Display

Figure 3: Schematics for our optical setup using: (a) an abstract light-field display; (b) a dual-stack of LCDs and (c) a lenticular array. (a) the light-field setup is placed at a distance t from the eye, which has axial length (size of the eyeball) equals to a. A light-field ray from $S_{2}$ at an angle $\alpha$ reaches a retinal location $R$ through a corneal point $k$. The focal length $f(k)$ comes from the eye's wavefront map, given as input, and drives the refraction of the light ray. (b) a dual stack of LCDs, placed $m$ apart, implements a light-field display by showing patterns on $L C D_{1}$ and blocking or allowing desired light rays on $L C D_{2}$. In this example, the setup creates two image planes $I_{k_{1}}$ and $I_{k_{2}}$ (colored dots) to compensate for differences in focal lengths from points $k_{1}$ and $k_{2}$ in the cornea. Refraction of $k_{1}$ is stronger than $k_{2}\left(f\left(k_{1}\right)<f\left(k_{2}\right)\right)$. The distance $j_{k_{1}}$ points out the depth at which a point conjugates to the retina when going through $k_{1} . j$ can be seen as the depth at which the subject is focusing, but varying over the cornea. Image planes $I_{k_{i}}$ are projected at the respective $j_{k_{i}}$ distances from the eye. $j$ also drives a magnification component $\left(-a / j_{k}\right)$ : the virtual image $I_{k_{1}}$ (colored dots) is smaller than $I_{k_{2}}$. Each colored dot has its own rays being cast for specific sections on the eye. (c) an LCD with a lenticular overlay implements a light-field display by drawing patterns on LCD $D_{1}$ and directing light rays from the lenticular. A collimated bundle of light rays of angle $\alpha$ is focused by a wide region on the eye lens onto $R$. Variations of focal length inside the red-dot-stroked region $\left(k_{0} . . k_{n}\right)$ blurs $R$. In this scenario, $m$ is one lenslet focal length from $L C D_{1}$.

ity by adding corrective lenses; (ii) retinoscopy, where skilled optometrists check for the reflected light slit into the retina; (iii) NETRA, which uses high-resolution light fields and interactive techniques to self-evaluate refractive errors [Pamplona et al. 2010]; (iv) auto-refractors for automated Scheiner tests [Porterfield 1759]; and (v) Shack-Hartmann sensors to compute distortions observed in a known light pattern [Liang et al. 1994]. Solutions (i-iv) result in measurements for $S, C$ and $\gamma$ parameters while (v) results in a wavefront map. Both formats are accepted inputs to our approach.

Cataracts are light scattering proteins that opacify the lens in certain regions, blurring the eye's point spread function. Measuring techniques include: (i) slit-lamp microscope to capture backscattered light; (ii) Scheimpflug photography to shear the camera's depth of field and capture sharp images from the cornea to the posterior lens capsule; (iii) retro-illumination reflects light from the retina and captures cataracts as blurry blobs; (iv) Shack-Hartmann [Donnelly et al. 2004] casts coherent light rays, hitting cataracts and reaching a light-field sensor as blurred spots; (v) CATRA scans the lens, section by section, to map opacities as seen by the viewer [Pamplona et al. 2011]. We use cataract density maps to avoid light paths.

Although the majority of this paper deals with focusing range, opacities, and aberrations in the human eye, the reader can see the eye as a camera with aberrated lenses. Terminology, diagrams and analysis are intentionally similar to traditional camera and rendering materials in graphics. For sake of simplicity, the discussion uses $2 \mathrm{D}$ geometric optics (1D sensor and $2 \mathrm{D}$ ray space) but it applies to $3 \mathrm{D}$ without major changes. Although derivations use a single intensity value per image pixel, equations can be independently applied to red, green and blue channels of input color images.

\section{Building Tailored Light Fields}

Our approach can be described as the projection of depth-dependent anisotropic patterns according to the spatially-distributed optical aberrations of the eye. These optical aberrations are represented as focal lengths on the wavefront map. The depth-dependent patterns are anisotropic images virtually placed at the right point in focus for a given optical power on the wavefront map. Figure 3(b) shows two image planes $\left(I_{k_{1}}\right.$ and $\left.I_{k_{2}}\right)$, each one for a given corneal point $\left(k_{1}\right.$ and $k_{2}$ ). Since the optical power of $k_{1}$ is stronger than $k_{2}, I_{k_{1}}$ must be placed closer and magnified accordingly. Notice how the individual light rays from respective objects on each image plane are integrated on the retina. Because these images are placed at multiple depths to create a single image in focus, we say our system has a multi-depth feature. Our method breaks an object's light field into several parts and virtually places them into these depths, making sure they are seen only through eye sub-apertures with given refractive powers. Light paths that go through opacities or unpredictable scattering sites, such as cataracts, are avoided. The final result is a light field to be displayed at a distance $t$ from the eye.

We call tailoring the process of adapting a light field to compensate for an individual's inability to focus. It is performed in two main steps: (i) pairing light-field rays $\left(S_{2}, k\right)$ and retinal positions $(R)$ to associate a raw intensity to each ray; and (ii) normalizing retinal "pixels" to avoid higher intensities in the image center, and noise due to a irregular discretization of the pupil area. Given as input an expected image on the retina $\left(I_{\text {Retina }}\right)$ and wavefront and cataract maps of the subject's eye, the method produces a light field to be shown on a specified display. Using geometric optics and thin lens model, the relation between a ray from point $S_{2}$ on the display at an angle $\alpha$ and the retinal point $R$ is given by (Figure 3(a)):

$$
\begin{aligned}
k & =S_{2}+\tan \alpha t \\
R\left(S_{2}, k\right) & =a\left(\frac{-k}{f(k)}+\tan \alpha\right)+k,
\end{aligned}
$$

where $f(k)$ is the focal length at position $k$ on the eye's aperture, $t$ is the distance from the light-field display to the eye, and $a$ is the eye's axial length. $f(k)$ is computed from the wavefront map or through the interpolation of the user's prescription data (Section 2). $a$ is essentially a scaling factor, measured to a specific viewer (alternatively, one can use $23.8 \mathrm{~mm}$, which is the average value of a human eye axial length [Schwiegerling 2004]). Energy $I$ reaching the retina at point $R$ is the integral of the incoming energy through all corneal points visible through a pupil with diameter $p$ :

$$
I_{\text {Retina }}(R)=\int_{-p / 2}^{p / 2} I_{\text {Lightfield }}\left[S_{2}(R, k), k\right] h(k) \mathrm{d} k,
$$

where function $S_{2}(k, R)$ is obtained by solving Equation 1 for $S_{2}$. Values must be clipped afterwards to the spatial limits of the dis- 
play. $I_{\text {Retina }}(R)$ is the accumulated intensity at point $R$ on the retina. $I_{\text {Lightfield }}\left(S_{2}, k\right)$ is the intensity delivered by the light field through the corneal point $k$ from position $S_{2}$ (Figure 3(a)). $h(k)$ is a binary visibility function for medium opacities (cataracts).

A trivial solution for Equation 2 can be obtained by having a single light ray from $S_{2}$ to each retinal point $R$. The integral over the pupil would be removed, only one $k$, say $k_{1}$ on Figure 3(b), would be used to create a one-to-one mapping. There would be only one image plane $I_{k_{1}}$ and the subject would be able to see it sharply. Although this would work, a huge amount of light would be required to see the displayed image. The use of many light-field rays to form each pixel on the retina (many-to-one mapping) enhances brightness, but requires a normalization step. The intensity of a light-field ray is then the retinal intensity divided by the number of incoming rays $n(R)$ at each retinal position $R$ :

$$
I_{\text {Lightfield }}\left(S_{2}, k\right)=\frac{I_{\text {Retina }}(R)}{n(R)} .
$$

Cataracts Although the point spread function of a cataract can be measured, cataracts make it hard to predict where individual light rays fall on the retina. We remove cataract-affected areas from the tailoring procedure by using a binary function $h(k)$ on Equation 2 , which is based on the cataract density function $c(k)$ [Pamplona et al. 2011] and a threshold $H$. The threshold sets the cataract density at which effects stop being noticeable:

$$
h(k)= \begin{cases}1 & \text { if } c(k)<H \\ 0 & \text { if } c(k) \geq H\end{cases}
$$

Green circles on Figure 4(e) and (f) highlight where light rays are being blocked because of $h(k)$.

Accommodation Tailored displays are used outside an individual's focal range, which changes with visual aberration (Figure 2). While multi-focus displays are mainly concerned with creating illusions realistic enough to make the subject accommodate to a given depth, a tailored display only adjusts itself to project images where the subject is already focusing. Focal lengths on a standard wavefront map $f_{\text {map }}$ are captured with zero accommodation power $(A=0 D)$. Since convergence rules accommodation [Maddox 1886], we assume the overall environment makes the subject accommodate at the closest possible point on her focal range to the display. When a hyperopic subject converges to a close-by display, she accommodates to focus at the display. Since she cannot focus very close, her accommodation stops at the closest possible point of focus, behind the display. For a myopic individual, if she converges to a far billboard, her accommodation follows convergence and relaxes the eye up to the most distant point of focus, which happens to be before the billboard. The focal length $f$ required for Equation 1 is then $1 / f=1 / f_{\text {map }}+A$, where $A$ :

$$
A= \begin{cases}0, & \text { if } t>z_{\text {farthest }} \\ \frac{1}{z_{\text {closest }}}-\frac{1}{z_{\text {farthest }}}, & \text { if } t<z_{\text {closest }} \\ \frac{1}{z_{\text {focus }}}-\frac{1}{z_{\text {farthest }}}, & \text { otherwise. }\end{cases}
$$

where, $z_{\text {closest }}, z_{\text {farthest }}$, and $z_{\text {focus }}$ are respectively, the closest, farthest and the current point in focus for a subject. Nearsighted subjects (first case) have their eyes relaxed when looking at the display. Farsighted ones (second case) are using full accommodative power. In the third case, since the display is inside the accommodation range $\left(z_{\text {closest }}<t<z_{\text {farthest }}\right), f$ is adjusted to the current eye focal length $\left(z_{\text {focus }}\right)$. In this case, one can disable all the computation and use a normal display instead.

Matrix Notation $\quad a$ and $t$ are setup constants while $f(k)$ is fairly steady for each corneal position $k_{i}$ over time. Equation 1 , the $S_{2}-R$ relation, can be re-written as an affine transformation (Figure 3(a)):

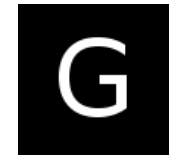

(a) Input

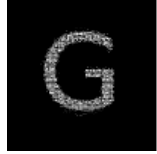

(b) Simulation

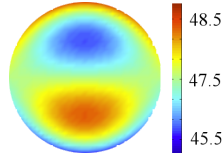

(c) Wavefront Map

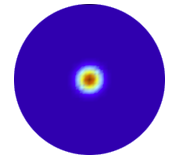

(d) Cataracts

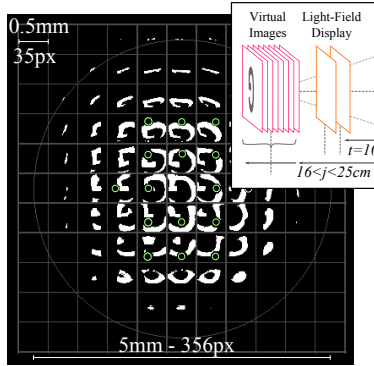

(e) $L C D_{1}$-Lenslets

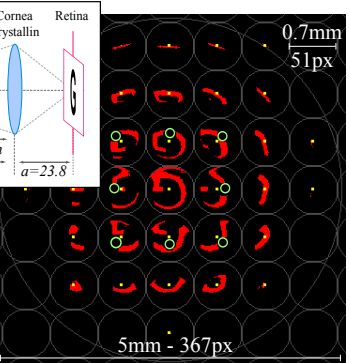

(f) LCD Stack
Figure 4: Simulation for the letter $\mathbf{G}(a)$ with height of $\approx 0.90 \mathrm{~mm}$ on the retina of a $2 D$-coma, $+5 D$-myopia (c), and cataractaffected $(d)$ subject. $\mathbf{G}$ is virtualized at many depths $(16 \mathrm{~cm}<$ $j<25 \mathrm{~cm}$ ). (b) is a software simulation of the composed image reaching the subject's retina. (c) Wavefront aberrations best fitted with Zernike polynomials. A standard emmetrope has optical power of $\approx 42 D$ (infinity conjugated with the retina), plus $+5 D$ of myopia takes it to $47 \mathrm{D}$ on average. Coma (blue and red regions on (c)) makes the refractive power change $\pm 1 D$, from $46 D$ to $48 D$. (d) is a scattering density map, red means less than $50 \%$ of light passing through. (e) shows $L C D_{1}$ for a lenticular based prototype and $(f)$ shows $L C D_{1}$ as red and $L C D_{2}$ as yellow for a dual stack of LCDs. Small quads in (e) mark the lenslets position and small gray circles in $(f)$ mark its cross-talk limits. Giant gray circle reflects a $5 \mathrm{~mm}$-diameter pupil. Notice how the letter is deformed behind each lenslet. Greenish circles in $(e)$ and $(f)$ highlight the cataract-avoiding feature of our algorithm. For these renderings: $t=10 \mathrm{~cm}, m=13.8 \mathrm{~mm}$ and $a=23.8 \mathrm{~mm}$. Retinal image (a) is magnified by $M \approx 8.4$ to $7.56 \mathrm{~mm}$ in height at a distance $j=20 \mathrm{~cm}$ from the eye. Pinhole pitches $(0.5 \mathrm{~mm}$ and $0.7 \mathrm{~mm})$ are not far from the pixel pitch of a $90 \mathrm{DPI}$ display $(0.3 \mathrm{~mm})$.

$$
\begin{aligned}
R\left(S_{2}, k\right) & =\frac{-a}{t} S_{2}+\left(\frac{-a}{f(k)}+\frac{a}{t}+1\right) k \\
R\left(S_{2}, k\right) & =M S_{2}+B_{k},
\end{aligned}
$$

where magnification $M=-a / t$ is a constant slope (assuming $a$ is the standard eye axial length), independent of the subject's refractive errors. In matrix notation, the light-field computation is:

$$
\left[\begin{array}{ccc}
R_{1, k_{1}} & \cdots & R_{r, k_{1}} \\
R_{1, k_{2}} & \cdots & R_{r, k_{2}} \\
\vdots & \ddots & \vdots \\
R_{1, k_{n}} & \cdots & R_{r, k_{n}}
\end{array}\right]=\left[\begin{array}{cc}
M & B_{k_{1}} \\
M & B_{k_{2}} \\
\vdots & \vdots \\
M & B_{k_{n}}
\end{array}\right]\left[\begin{array}{ccc}
S_{2_{1}} & \ldots & S_{2_{r}} \\
1 & \cdots & 1
\end{array}\right],
$$

where $r$ is the number of spatial samples on the light field and $B_{k_{i}}$ is the y-intercept value from Equation 7 for each sample $k_{i}$ on the eye's aperture (i.e., pupil). Wavefront maps, as captured by currently available Shack-Hartman systems, sample the aperture in the order of a couple of thousands points. Equation 8 is point-wise, making it suitable for GPU data-parallel computation. Since the overall non-linear mapping plus normalization is image independent, it can be pre-computed for a given subject's refractive errors, position and viewing direction.

Implementation Given a light-field display setup, wavefront maps $f(k)$, and cataract maps $h(k)$, our method computes the position $R$ for every pair $\left(S_{2}, k\right)$ by applying Equation 8 . Number of 


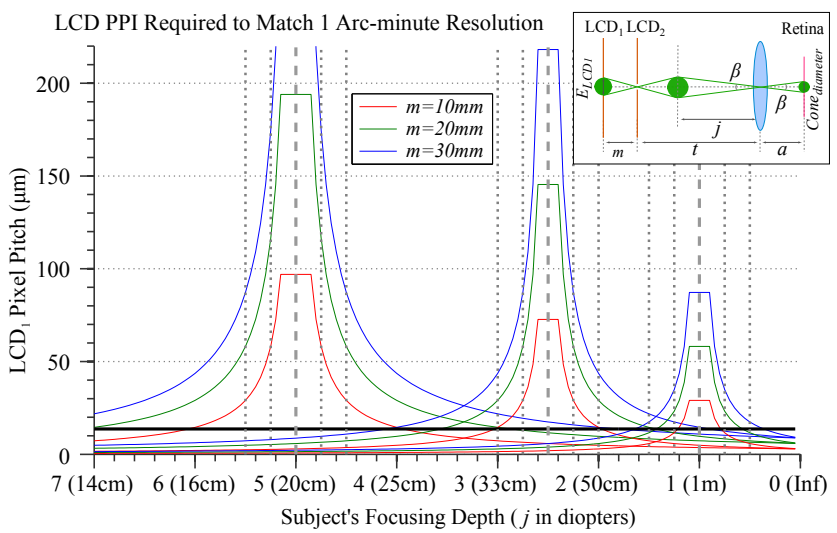

Figure 5: Pixel pitch required to create virtual images that match the human 1 arc-minute retinal resolution is currently available in non-expensive LCDs. Figure shows nine tailored displays (peaks) positioned at $t=[0.2 \mathrm{~m}, 0.4 \mathrm{~m}, 1 \mathrm{~m}]$ (bold dashed vertical lines), with $\mathrm{m}=[10 \mathrm{~mm}, 20 \mathrm{~mm}, 30 \mathrm{~mm}]$ as red, green and blue for each display. Each curve shows the required pixel pitch on $L C D_{1}$ for focal points $j$ (Equation 9). As the subject focus $j$ moves away from the display depth $t$, the required pixel pitch for $L C D_{1}$ increases. Thinner dotted vertical lines highlight the subject focus when close the display, in steps of .25D. The horizontal line is the resolution of our evaluation prototype (1857 PPI). For focal depths above this line, the display supports virtual images into the eye's resolution. Peaks are cropped when $|1 / t-1 / j|<0.2 D$. As $m$ grows and/or $t$ decreases, cheaper $L C D$ panels can be used.

accesses to each retinal point $R$ are computed and stored. Given a desired retinal image $I_{\text {Retina }}$, we set $I_{\text {Lightfield }}\left(S_{2}, k\right)$ from $I_{\text {Retina }}(R)$ and apply Equation 3 to normalize the intensity of each ray defined by $(S 2, k)$. Light-field setups can be built using a dual stack of LCDs or with an LCD plus a lenticular array. Ray discretization and the computation direction is technology driven. To avoid cross-talk, the dual stack of LCDs uses forward computation, from the light field to the retina, and $\left(S_{2}, k\right)$ is discretized according to pixel pitch of both LCDs. Lenticular setups, on the other hand, use backward computation (from the retina and cornea to the light field), and are discretized on the $I_{\text {Retina }}$ pixel pitch and the wavefront maps. Figure 4 shows how a computed light field looks like for a dual stack of LCDs (f) and with lenticular arrays (e). Further details are discussed on Sections 4 and 5.

Color Using color images simply require applying Equations 1 or 8 per color channel. Since the pixel pattern must be taken into account, the striped pattern used on standard LCDs is preferred. Horizontal positions are shifted in steps of a third of a pixel to compensate for displacements on the panel. Since refraction is wavelengthdependent (2 diopters from $400 \mathrm{~nm}$ to $800 \mathrm{~nm}$ [Campbell 2010]), one would expect to have different corrections for each color channel. However, the refractive variations among wavelengths also occur naturally in the eye and they provide cues to the accommodative procedure. The eye focuses on a specific wavelength, which changes from subject to subject. Colors allow the accommodation process to sample two or more image planes and foresee the direction of focus [Rucker and Kruger 2006]. Although a tailored correction per wavelength may increase visual acuity, to our knowledge, the eye response to this new stimulus is unknown. We also notice that color channels are not single wavelength but an overlapping group of wavelengths with different profiles among manufacturers. In our tests, we do not perform any additional color calibration; rather, we rely upon the manufacturer supplied color calibration.

System Alignment is required only when correcting high-order

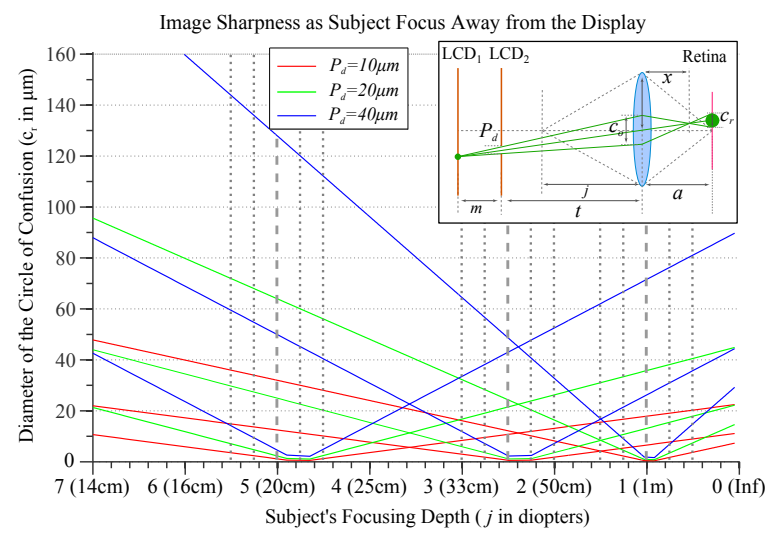

Figure 6: Diameter of the circle of confusion $c_{r}$ created on the retina as a function of where the subject is focusing $(j)$. Three pinhole sizes $P_{d}$ are shown as red, green, and blue lines. Each circle of confusion line is plotted for three displays using $m=$ $[10 \mathrm{~mm}, 20 \mathrm{~mm}, 30 \mathrm{~mm}]$ and $t=[20 \mathrm{~cm}, 40 \mathrm{~cm}, 1 \mathrm{~m}]($ bold dashed vertical lines). As the subject focus moves away from the display, the circle of confusion grows. The growth rate is defined by $P_{d}$.

aberrations. For spherical aberrations (farsightedness, nearsightedness and presbyopia), $f(k)$ is constant across the cornea. As the subject's head shifts, the retinal image translates while the integration of light-field pieces on the retina remains aligned. Astigmatism does not require translational, but a rotational alignment. Misplacing the corrected angle of astigmatism $(\gamma)$ creates undesirable blurriness proportional to the cylindrical power $(C)$. For higher-order aberrations and cataracts, alignment is critical. Just like glassesfree 3D displays, subjects must keep the computed position. The same way one searches for the best angle to see the $3 \mathrm{D}$ effect, subjects naturally search for the best angle to see our images clearly. Real-time eye-tracking systems could improve this experience.

Resolution System resolution is dependent on the angular resolution of the light field, which is defined by the distance $m$, between LCDs or LCD-lenticular, and by the pixel pitch of $L C D_{1}$. Resolution of the system is given by pixel arc-minute resolution of depthdependent virtual images $\left(I_{k}\right)$ as they move away from the display. The required $L C D_{1}$ pixel pitch to match 1-arc minute resolution on the retina is available on today's technology (Figure 5). The lightfield distribution can be placed a few diopters off the screen while their pixel pitch is still into the retinal visual acuity. Virtual images are magnified by $M=-a / j$ on the retina. Behind each pinhole, segments of the virtual image are magnified by $m /(t-j)$, where $j$ is the distance from the virtual image to the eye and computed by $1 / j=1 / f(k)-1 / a$. To match an individual's retinal resolution $\beta$ (e.g., 1 arc minute), $L C D_{1}$ should have pixel pitch $\left(E_{L C D_{1}}\right)$ of:

$$
E_{L C D_{1}}=\frac{m j \tan \beta}{t-j}
$$

Figure 5 shows a plot for Equation 9. The best setup parameters are application dependent. For instance, a monitor placed $t=5 \mathrm{~m}$ from the eye requires larger angular resolution - bigger $m$ - compared to an e-book reader, which instead, can have smaller $m$. Although Figure 5 is computed for the best perceivable image ( 1 arc-minute resolution), we notice that the display does not need to perfectly match it. For instance, the uncorrected visual acuity of a slightly nearsighted subject $(+1 D)$ is $\approx 13$ arc minutes. A display that corrects it to 3 or 2 arc minutes improves her visual acuity by $80 \%$.

Display Size An image displayed at a monitor with diagonal of $w=23$ " requires a light-field display with roughly the same size: $w^{\prime}=w-p m / t$, where $p$ is the pupil diameter. Refractive er- 
rors add variability to the spatial coverage, but they are insignificant compared to the image size. The distribution of light among covered pixels follows a quadratic decay when reaching the display border. To minimize this decay, the light-field display size must be:

$$
w^{\prime}(w)=\max _{k}\left(\left|\frac{w}{2 t} \frac{-k}{j} 2(t+m)+k\right|\right),
$$

where $1 / j=1 / f(k)-1 / a$. Additional brightness decay is caused by the tilt on the available cone of light. Each pixel on the virtual image plane $I_{k}$ is a cone vertex connected to the pupil border. Pupil and the light-field display planes are conical sections with variable area, which are circular when the vertex is on the optical axis, and elliptical when off axis. As the cone vertex moves away from the optical axis, keeping the same depth $j$, conical sections decrease in size, making the borders of the image dimmer by gathering less light from the light field. Both decays are normalized, with loss of brightness, by Equation 3 .

\section{Dual LCD-Stack Tailored Display}

One method to implement a light-field display using off-the-shelf hardware is to stack two LCDs displays. A first LCD shows our distorted patterns while a second LCD blocks light in specific directions. Known as parallax barriers [Ives 1903; Isono et al. 1993], these hardware setups coupled with smart algorithms are typically used to create 3D stereoscopy by projecting images for the left and right eyes. These algorithms, however, do not account for the subject's eye parameters: focal point, low and higher-order aberrations, and cataracts. Our solution uses a similar hardware setup but also warps the pattern as needed to additionally support the aforementioned effects. A matrix of deformed sections of the desired retinal image is displayed on $L C D_{1} . L C D_{2}$ acts as a pinhole filter to position these sections properly on the retina. The warping transformation is different for each pinhole (Figure 4(e) and (f)). For low-order aberrations (nearsightedness, farsightedness, and presbyopia), changing pattern's position and size is enough to make it converge on the retina. For astigmatism, cataract and higher-order aberrations, however, the pattern itself must deform.

On the dual-stacked-LCD setup, the outgoing ray angle is given by $\tan \alpha=\left(S_{2}-S_{1}\right) / m$ (Figure 3(b)). Pixel intensities on $L C D_{1}$ are given by Equation 3, while pixels (pinholes) on $L C D_{2}$ are set to maximum intensity. The energy $I$ reaching $R$ is given by the sum of the product of LCD pixel intensities going through the cornea:

$$
I_{\text {Retina }}(R)=\sum_{k=-p / 2}^{p / 2} I_{L C D_{1}}\left[S_{1}(k, R)\right] I_{L C D_{2}}\left[S_{2}(k, R)\right] h(k) .
$$

In practice, the discretization of the LCD pixels creates light rays with an area equivalent to the pixel size of $L C D_{1}$. As the pattern deforms, more energy is concentrated on shrunk parts that will be spread out onto bigger sections on the retina. These parts must emit more energy to keep the brightness once on the retina. The intensity of each light ray is the sum of energies over the area it represents on the retina normalized by the number of incoming rays $n(R)$ at each retinal position $R$ :

$$
I_{L C D_{1}}\left(S_{2}, k\right)=\int_{R-q}^{R+q} \frac{I_{\text {Retina }}(i)}{n(i)} \mathrm{d} i,
$$

where $q$ is the equivalent radius of a single light-field pixel on the retina computed by:

$$
q=E_{L C D_{1}} \frac{a(t-j)}{m j},
$$

where $E_{L C D_{1}}$ is the pixel pitch/size of $L C D_{1}$. To avoid repeatedly evaluating the integral, one can use a summed area table to accumulate the intensities of the retinal image pixels.

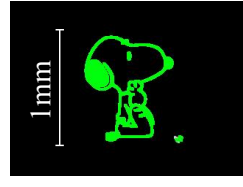

(a) Input

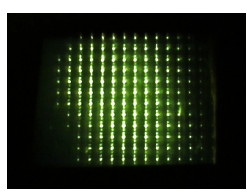

(d) Emmetrope

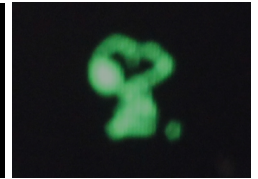

(b) Regular Display

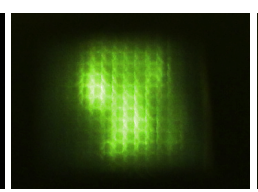

(e) Myopia 3.00D

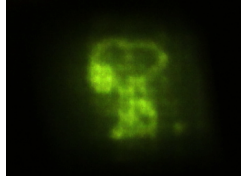

(c) Tailored Display

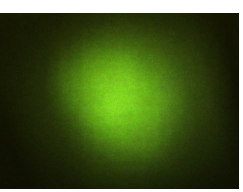

(f) Myopia 3.75D

Figure 7: Picture (c) reproduces the perception of (a), tailored for a 3.25D myopic vision. As a comparison, (b) shows how the subject will see the image at a regular display. The tailored image plane is at subject's farthest focal point $(j=30.7 \mathrm{~cm})$ and the display is placed $t=37 \mathrm{~cm}$ away from the eye $(0.5 \mathrm{D}$ difference). This figure shows how precise our prototype is for very small focal changes of small images. To simulate this myopic perception, we place $a+3.25 D$ optometric lens in front of the camera's aperture focusing at infinity. The bottom row shows how subjects that do not match the tailored image perceive it: (d) an emmetrope focusing at the display; (e) a 3D-myope (slight deviation from the tailored one) which focus after the tailored image depth $(33.3 \mathrm{~cm})$ and $(f)$ a $3.75 D$-myope which focus before the tailored image depth $(26.3 \mathrm{~cm})$. The 150-px-tall image $(a)$ is $\approx 1 \mathrm{~mm}$ tall on the sensor and is magnified by $j / a \approx 5.6 \mathrm{~mm}$ at the focal point. Pixel pitch at the virtual image plane is $\approx 37.3 \mu \mathrm{m}$ ( 0.4 arc minute). Pinholes have area of $3 \times 3$ pixels $\left(P_{d}=42 \mu \mathrm{m}\right)$. This test uses small inputs to reach the limits of acuity: $0.55 \%$ of the picture area.

Avoiding Cross-talk The presence of cross-talk creates ghost images, random noise, and requires additional computation to renormalize energies on the retina. We avoid cross-talk by setting a minimum pinhole pitch (i.e., the separation between two adjacent pinholes) $P_{p}=p m / t$ of $L C D_{2}$, where $p$ is the aperture (pupil) diameter. Since most setups have working distance $t>20 \mathrm{~cm}$ (and hence $t \gg m$ ) and the pupil diameter is $9 \mathrm{~mm}$ at most, the possible cross-talk area on $L C D_{1}$ is naturally quite small. Pinhole pitch is usually smaller than $1 \mathrm{~mm}$, close to a 90-PPI display pixel pitch.

Sharpness Analysis The sharpness of the resulting imagery is measured by the size of the circle of confusion $c_{r}$ on the retinal plane, which depends on the pinhole diameter $P_{d}$ (Figure 6(top)). The diameter of the cone of confusion $c_{o}$ on the corneal plane is given by $c_{o}=t P_{d} / m$ (assuming a locally flat cornea). When $f(k)$ is constant inside $c_{o}$, the circle of confusion mimics the one from an object at $L C D_{1}$, distance $t+m$ from the eye (Figure 6). Then $c_{r}=c_{o}(a-x) / x$, where $x$ is the distance to the conjugate plane for $L C D_{1}$. Assuming a thin lens system: $1 / x=1 / f(k)-1 /(t+m)$.

\section{Lenticular-based Tailored Display}

In this setup, $L C D_{2}$ is replaced by a microlens array. Compared to the dual-stacked $\mathrm{LCD}$, it provides wider apertures for $L C \mathrm{D}_{2}$. The eye captures more light, enhancing brightness while preserving sharp images. Instead of a single ray coming from each pinhole in one direction, the eye lens converges a bundle of rays (Figure 3(c)). When properly designed, the entire bundle is focused into a single point on the retina. The relation among $S_{1}$ and $R$ follows Equation 1 , but $\tan \alpha=\left(L_{c}-S_{1}\right) / m$ where $L_{c}$ is the lenslet center.

As a bundle of light rays reaches a sector on the cornea (red ellipse 


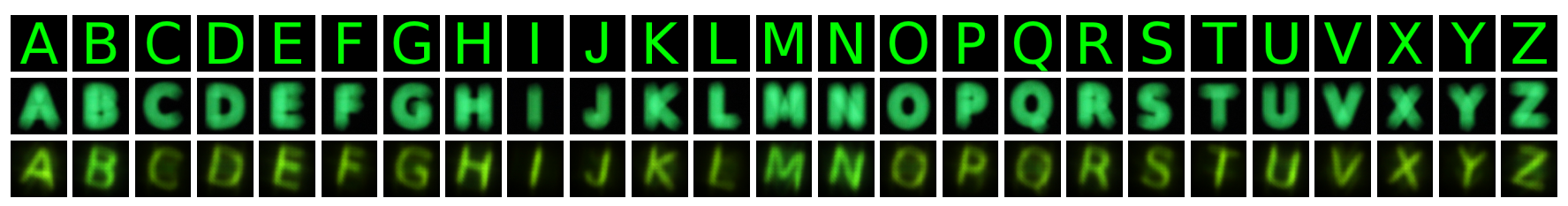

Figure 8: (top) Input images to the tailoring process. (middle) Simulated perception by a presbyopic subject of the respective target letters using a regular display. (bottom) Observation of the respective pre-distorted target letters but shown by our tailored display for the same presbyopic aberration. Subject's closest focal point is at $1.6 \mathrm{D}(\approx 60 \mathrm{~cm}$, behind the display). Camera is placed $38.5 \mathrm{~cm}$ away from the display. $p=28 \mathrm{~mm}, m=10.7 \mathrm{~mm}, a=70 \mathrm{~mm}$. Images are $1.41 \mathrm{~mm}$ tall on the retina, reaching $\approx 12.6 \mathrm{~mm}$ on the virtual image plane. Pixel pitch at the virtual image is $0.125 \mathrm{~mm}$ (0.69 arc minute resolution). Hardware misalignment produced slanted letters.

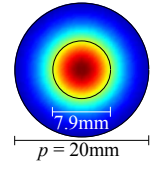

(a) Occlusion

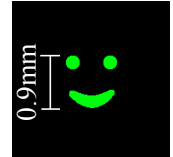

(b) Input

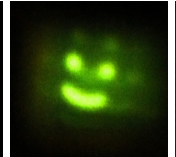

(c) Clean lens

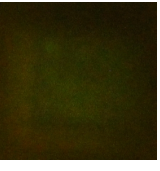

(d) Cataracts

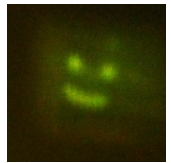

(e) Tailored

Figure 9: Cataract affected camera $(a)$ sees the green smile $(b)$ without correction (d) and with tailored correction (e). The normalized cataract density map (a) with a threshold of 0.4 (inner circle) is used as input. Rays reaching the sensor through the inner circle are removed. (c) how a non-cataract-affected camera would see (b). (d) decreases on intensity due to the use of pupil borders only. To take the picture we added an $8 \mathrm{~mm}$-tall diffuser on top of the camera lens. Here $t=47 \mathrm{~cm}$ and resolution of 0.87 arc minutes.

on Figure 3(c)) and focus onto $R$, the lenslet pitch must match a corresponding sector of the eye with constant optical power. Variations on refractive power inside the corneal area touched by the beam creates blur. An optimal beam diameter (lenslet pitch) must expand to a corneal area with variations smaller than $0.16 D$ (i.e., unnoticeable blur for 1-arc minute visual acuity). Since refractive aberrations are smooth and continuous, and the sample pitch of a high-quality Shack-Hartmann device reaches $209 \mu \mathrm{m}$ on the cornea [Rozema et al. 2005], beam sizes about $200 \mu \mathrm{m}$ are expected to create minimum blur, if any. $200 \mu \mathrm{m}$ microlens pitch is available by many lens arrays manufacturers today.

Bundle of light rays introduces another source of blur called focus ambiguity. The eye focuses at the projected image $(j)$ and not at the depth the bundle represents. For instance, if $m$ is equal to the lenslet focal length $\left(f_{L}\right)$, the bundle of light rays becomes parallel (Figure 3(c)), simulating an object at optical infinity. If the sector of the eye aperture that receives this bundle is not focusing at infinity, the point becomes blurred on the retina. In the optimal scenario, each bundle must represent an object depth at $j$, which is dependent of the focal length $f(k)$ of the corresponding corneal area:

$$
\frac{1}{m}=\frac{1}{f_{L}}+\frac{1}{j-t}
$$

where $f_{L}$ is the lenslet focal length and $m$ the distance between $L C D_{1}$ and the lenticular array. Since $m$ is a parameter of our tailored display (constant for a given prototype), one can adjust it to minimize the blur for a few expected spherical cases (myopia, hyperopia and presbyopia). In the case of a tailored television, placed at $t \approx 5 m$ from the eye, $m$ would be very close to $f_{L}$ since the first term dominates Equation 13. A tailored e-book reader, placed $t=20 \mathrm{~cm}$ from the eye, would have $m<f_{L}$, when $j>t$ (fixing for farsightedness), and $m>f_{L}$, otherwise (fixing for very strong nearsightedness: $>5 D$ of myopia). As $j$ approaches $t$, the optimal $m$ becomes more significant. This trick cannot create images when $j=t$. We used the same value of $m$ in all evaluations. Optimizing $m$ for a given device is useful to drive product designers to achieve the sharpest result when using the lenslet array approach and cor-

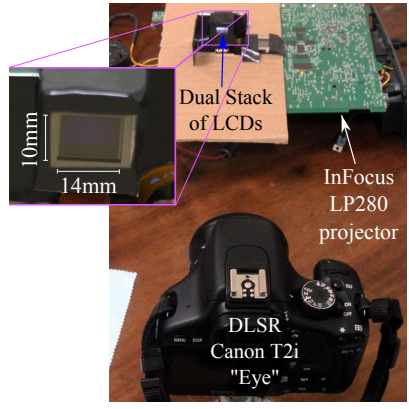

(a) Dual-LCD Projector

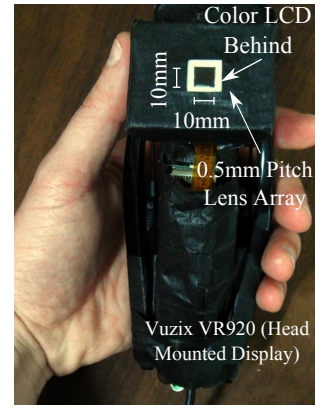

(b) Lenticular HMD
Figure 10: Two of our prototypes:(a) projector-based monochrome lcd-stacked display, and (b) head-mounted display with a lenticular array. Both LCDs have $\approx 1,800$ PPI and measure about $14 \times 10 \mathrm{~mm}$. The spatial resolution is limited by the small screen size. An array of $L C D$ s is required to replace a standard monitor. The angular resolution, however, nicely fits to tailoring purposes.

recting spherical aberrations only. After the device is built, $m$ does not change.

In case of astigmatism, cataracts, and higher-order aberrations, $m$ cannot change among sub-apertures. Also, the bundle simulates a virtual object through a cone of light that expands its radius as the virtual object depth grows, reaching a bigger section on the cornea which may not have constant optical power (like the spherical cases). We opted for having $m$ equal to the lenslet focal length $f_{L}$, since beam radius at the corneal plane is constant to the lenslet pitch. The circle of confusion is dependent on the subject's focal point only, from zero when focusing at infinity and linearly reaching $\approx 100 \mu \mathrm{m}$ in extreme cases, such as myopia of $9 D$.

Avoiding Cross-talk Since lenslet pitch cannot be changed, lenslet focal length $f_{L}$ is carefully chosen to minimize crosstalk: $f_{L}=L_{p} t / p$, where $p$ is the pupil diameter and $L_{p}$ the lenslet pitch. One can cover all odd (or even) lenses on the array to reduce $m$ by half and quadruplicate the amount of pixels behind each lenslet.

Energy normalization For lenslet arrays, the energy $I$ reaching $R$ is the sum of rays through an integral over the lenslet pitch $L_{p}$ :

$$
I_{\text {Retina }}(R)=\sum_{k=-p / 2}^{p / 2} \int_{\frac{-L_{p}}{2}}^{\frac{L_{p}}{2}} I_{L C D_{1}}\left[S_{1}(k+z, R)\right] h(k+z) d z
$$

In practice, the integral is approximated by the product of the energy of the central light-ray $(z=0)$ by the beam area at the cornea. For spatially-variant refractive powers inside $k \pm L_{p} / 2$, like in bigger lenticular pitches, the integral must be computed. In these cases, the energy of a single pixel $S_{1}$ on $L C D_{1}$ reaches a retinal area around a central point $R . I_{L C D_{1}}\left(S_{1}\right)$ then is the sum of $I_{\text {Retina }}$ pixels inside the retinal area, each retinal sample divided by the number of rays reaching it. 


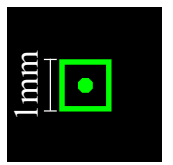

(a) Input

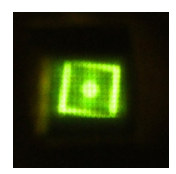

(b) Normal

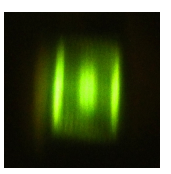

(c) Astigmatic

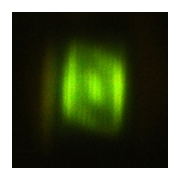

(d) Tailored

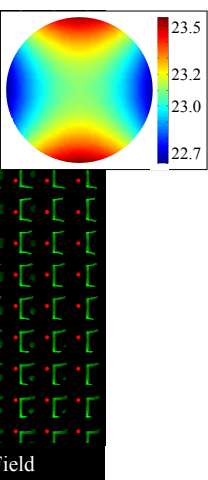

(e) Uncorrected and Tailored Light Fields

Figure 11: How an astigmatic subject sees the green square (a) without correction (c) and with tailored correction $(d)$. (b) shows how a non-astigmatic subject would see the same square. The light field for these pictures is shown on (e), not corrected on left and tailored on the right side. Pinholes in red. Notice how the pattern deforms under each pinhole. Astigmatic wavefront map has aberrations of $1 D$ in cylinder at the 180-degree meridian $(28<j<40 \mathrm{~cm})$. While an emmetropic view sees the effects of astigmatism (c), an $1 D$ astigmatic view perceives a corrected image $(d)$. Here $t=47 \mathrm{~cm}$. Resolution of 1.16 arc minutes.

\section{Prototypes and Evaluations}

Our dual-stack-LCD prototype uses components from an InFocus LP280 economy-class projector (Figure 10(a)). Two of the projector's monochrome LCDs (1, 857 pixels per inch - PPI) are stacked $m=10.8 \mathrm{~mm}$ apart with three polarizers positioned in front, in between, and behind them, respectively. A standard LCD light box is placed behind $L C D_{1}$. A Canon T2i DSLR to simulate eye aberrations is positioned towards the display with varying distance $t$. We add optometric lenses with known optical powers and/or diffusers in front of the camera to simulate refractive and scattering conditions. We also use a Vuzix iWear VR 920 head mounted display (HMD) for portable user testing (Figure 10(b)). Its 1,806PPI LCD is placed behind a 500-microns lenslets with focal length $12.5 \mathrm{~mm}$ (Edmund Optics NT64-479). To minimize cross-talk, we use a $3 \times 3$ subset of the lenses, $1 \mathrm{~mm}$ from each other (covering even lenses). Figure 4 shows how images for $L C D_{1}$ and $L C D_{2}$ look like when they are simultaneously corrected for myopia, cataracts, and coma in both setups. We evaluated our technique in three rounds: (i) on small binary images to investigate the suitability for inexpensive LCDs to produce sharp images at retinal resolution for a variety of eye conditions; (ii) on colored light fields to investigate how an array of such LCDs would be captured by the imaging system; (iii) by user evaluations to check the ability to correct images for human subjects. Our evaluation uses small images. Even a tiny deviation (i.e., $0.15 \mathrm{D}$ in our tests - Figure 7) is easily observed.

\subsection{Controlled Evaluations}

Nearsighted evaluation Figure 7(c) shows a picture from our dual-stack-LCD prototype correcting for a $3.25 D$ nearsighted vision. This subject requires eyeglasses of $-3.25 \mathrm{D}$ and has the farthest focal point at $j=30.7 \mathrm{~cm}$. The display is placed at $t=37 \mathrm{~cm}$
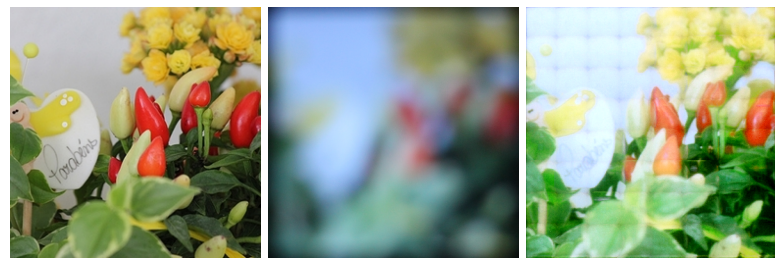

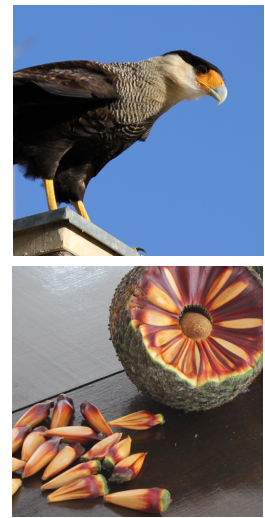

(a) Input Image

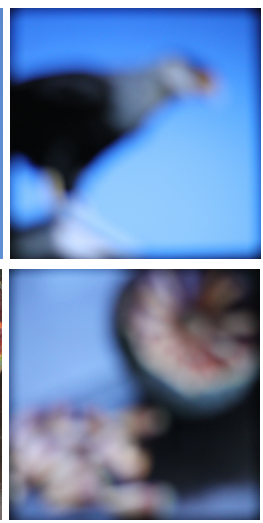

(b) Regular Display

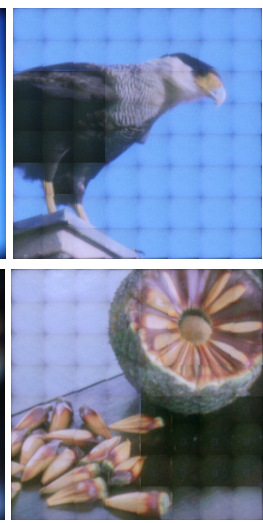

(c) Tailored Display
Figure 12: Input images (a) and how they are perceived on a tailored display by a farsighted subject (c). The HMD prototype was placed $t=20 \mathrm{~cm}$ from the eye (camera). The camera simulated a subject whose closest focal point is $j=50 \mathrm{~cm}$ (subject using $+3 D$ lenses). Picture (b) shows how the subject sees the respective image on (a), with same size, at the same distance in a standard monitor. Since our setups have small spatial resolution, we mimicked an array of LCDs by changing the image being displayed to cover a bigger "retinal" area. (b) is a collage (sum) of 64 square patches which create a 3.4 arc-minute image on the retina (each path $=0.425$ arc minutes). The blue channel of (c) was adjusted to $75 \%$ of its captured intensity to remove prototype light leaking.

from the eye. To simulate the optical distortion of this myopic subject, a camera is focused at infinity and we add a $+3.25 \mathrm{D}$ optometric lens in front of it. (b) shows how the same subject would see the image on a regular monitor. The test image is small $(1 \mathrm{~mm}$ on the retina). Pixel pitch at the virtual image plane is $\approx 37.2 \mu \mathrm{m}(0.4 \mathrm{arc}$ minute). Focusing the camera on the $L C D_{1}$, we simulate how an emmetrope (Figure 7(d)) sees the same scene.

Figure 7 also shows how a subject with $3 D$ and $3.75 D$ of myopia would perceive the light field corrected for $3.25 D$. Figure 7(e) and (f) reveal significant out-of-focus effects which intuitively do not agree with the common sense that 0.5 diopters is usually not noticed by most people. Although we are creating a correct image on the subject's focal point, out-of-focus blur is not expected to be equal to a regular one. The circle of confusion of a tailored illusion is a coded pattern and not a uniform distribution of energies. The small pattern enhances this effect, generating the overall blurriness of Figures 7(e) and (f). This evaluation with small images attest the precision of our setup, since small variations of 0.25 diopters strongly affect the visibility of the projected image.

Farsighted evaluation Using the same evaluation strategy, Figure 8 shows 25 letters as seen by a $1.6 \mathrm{D}$ presbyope (farsighted) individual, where the closest point in focus is $j \approx 60 \mathrm{~cm}$. This subject is required to use $+2 D$ or $+3 D$ on reading glasses. To take this picture, the camera was focusing at infinity and we inserted a $+1.5 \mathrm{D}$ lens in front of it. The virtual image is created behind the actual display and reaches 0.68 arc-minute resolution. Presbyopes and hyperopes are similar since both cannot focus close. 
Cataract evaluation Figure 9 shows how a cataract-affected camera (a scattering site of $8 \mathrm{~mm}$ diameter on the lens) sees a tailored light field according to Equation 4. This test also includes a $2.5 \mathrm{D}$ of myopia $(j=40 \mathrm{~cm})$. Figure 9 (a) shows the cataract density map. Light rays traveling inside the black circle are strongly scattered and create glare. Our method creates a light field that passes only through the borders of the aperture, outside the black circle, avoiding scattering. Again, the image is small, requiring the setup to be precise on tracing rays. Figure 9(d) shows how the cataract affected lens will perceive the pattern without correction. As a comparison, (c) shows how a myope-only subject will see the same light field. (e) shows how the cataract-affected camera sees our tailored light field. Since we are using the border of the lenses only, the brightness is diminished. Resolution of 0.87 arc minute.

Astigmatism evaluation Figure 11 corrects for a spherical power of $2.5 D$ of myopia plus astigmatism of $1 D$ at 180 degrees. This is a clear case where the virtual object spreads in space, from $j=28 \mathrm{~cm}$ to $j=40 \mathrm{~cm}$. The Zernike-fitted wavefront map shows the distribution of refractive aberrations over the eye's aperture. For the input image Figure 11(a), our method computed corrected ((e)right) and non-corrected ((e)-left) light fields. The pattern deforms under each pinhole. Figure 11(c) shows how an astigmatic subject would see a non-corrected light field. The square blurs in the vertical direction, following the strongest meridian in optical power. As a comparison, Figure 11(b) shows how emmetropes would see the uncorrected light field. Figure 11(d) shows our tailored light field for this eye condition. Although prototype alignment between the two LCDs blurred the horizontal lines of the image, one can see a squarish shape. Resolution of 1.16 arc minute.

Color evaluation Figure 12 validates our color extension with the HMD prototype. Images on column (a) are inputs to the method. We tailored light fields for a farsighted individual with closest point in focus at $j=50 \mathrm{~cm}$. The display is placed $t=20 \mathrm{~cm}$ from the eye (subject must use $+3 D$ reading glasses). Column (b) shows how the subject sees the respective image with same retinal size on a standard monitor at $t=20 \mathrm{~cm}$, the same distance of the tailored display. (c) shows how the subject sees our tailored light field. Since our LCDs have small size $\left(14 \times 10 \mathrm{~mm}^{2}\right)$, we mimicked an array of LCDs to cover a bigger retinal area. The grid-like effect results from the sum of images. Resolution for each image pixel is 0.43 arc minutes. Figure 1(top) compares the view of a 2.5 -diopter farsighted individual in regular and tailored displays. In this case, $t=38 \mathrm{~cm}$ and the subject can only focus at optical infinity (i.e. she had cataract surgery and her eyes do not accommodate).

Real-time Performance Our GPU implementation based on Equation 7 with color achieves real-time performance (140 frames per second) on a laptop with a GeForce 8400GS on a full $1280 \times 1024$ frame. The surprising suitability for tailoring videos without pre-processing is only surpassed by the intriguing notion that, if the wavefront aberration map could be measured instantly, our method could account for teardrop variations in real time.

\subsection{User Evaluations}

Spherical aberration 13 emmetropes (ages 21 to 27 , mean $24 \pm$ 1.88) simulate an exact $5 D$ of myopia by placing an optometric lens between their eyes and our HMD prototype. Software randomly shows 16 tailored light fields from $1 D$ to $5 D$ of myopia. Subjects must choose which view is better, with or without the lens, for every light field. As expected: (i) subjects have chosen the lens $98 \%$ of the time ( $\sigma=3 \%$ among subjects) when the light field tailored for $5 D$-myopia is shown (Figure 13); (ii) subjects have chosen to avoid the lens on $100 \%$ of cases when the light field was non-tailored. Virtual images used in the tests were projected from $j=20 \mathrm{~cm}$

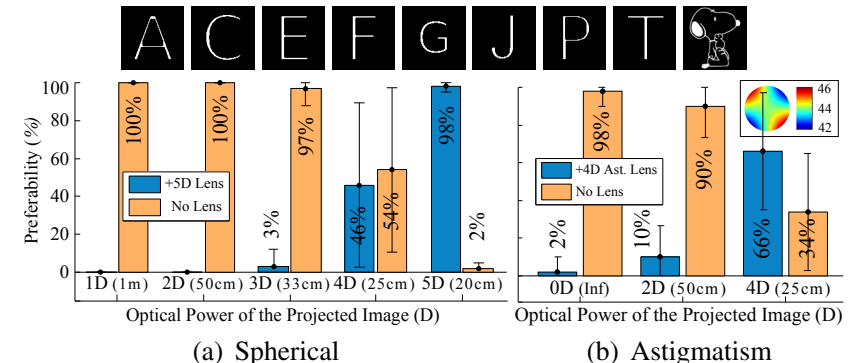

Figure 13: (a) 13 emmetropes voted for the best readable view, with or without a $+5 D$ lens (simulating $5 D$ myopia), for tailored images from $1 D$ to $5 D$ of myopia. As expected, images tailored for $5 D$-myopia are preferred to see through the $5 D$ lens. (b) 10 emmetropes voted for the sharpest view, with or without a $+4 D$ astigmatic lens. The HMD prototype is placed $t \approx 7 \mathrm{~cm}$ away from their eyes. A total of 189 votes were taken on (a) and 160 on (b). The 9 images on top were showed twice with different corrections. Error bars represent the standard deviation among people.

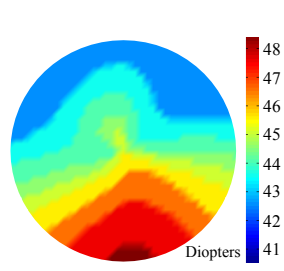

(a) Wavefront

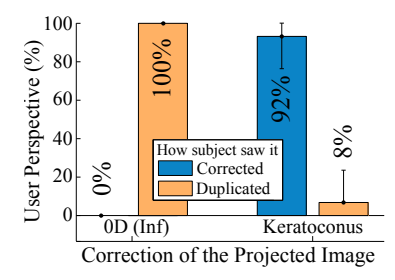

(b) Results
Figure 14: Subject with keratoconus (wavefront map in (a)) chooses which image is better: not corrected and tailored for his specific map. The view of a keratoconus-affected subject presents a duplication effect of bright objects. This effect was absent in $92 \%$ of the tailored images ( $\sigma=17 \%$ on a 5 round test). A total of 80 votes were computed for source images in Figure 13(top).

and $j=25 \mathrm{~cm}$ have resolution of $\approx 0.52$ and $\approx 0.42$ arc minute, respectively, below the standard eye acuity.

Intriguingly, user preference is not linear with the correction growth (Figure 13). For tailored light fields from $1 D$ to $3 D$, subjects clearly prefer no lens $(100 \%, 100 \%, 97 \%$, respectively). In our experience, accommodation takes place and makes subjects easily compensate for distortions of $1 D, 2 D$ and $3 D$. For $4 D$ cases, even though the accommodation power of most subjects goes up to $5 D$, the accommodation power required to keep a tailored image for $5 D$ in focus is too strong. When subjects add lenses, the eye relaxes and the preference for the lens grows. Heterogeneous focal ranges explain the bigger error bars for $4 D$.

Astigmatic aberration In the second evaluation, 10 emmetropes (ages 23 to 27, mean $24 \pm 1.6$ ) choose the best view between a naked eye and through an astigmatic lens. Software randomly corrects images for infinity $(0 D), 2 D$ and $4 D$ of astigmatism at 45 degrees. Subjects prefer the lens $66 \%$ of the time when the displayed image is corrected for the lens they were seen through $(\sigma=30 \%$ among subjects). We suspect some subjects focused on the spherical equivalent $\left(S_{e q}=S+C / 2\right)$ to overcome the blurriness generated by $2 D$ and $4 D$ corrected images when looking with naked eyes.

Keratoconus aberration In our last test, virtual images are tailored for a given keratoconic wavefront map (Figure 14(a)) captured from a Shack-Hartmann device. Keratoconus duplicates bright objects. Software randomly shows tailored and non-tailored light fields. A keratoconus-affected subject reports when he sees the 
double effect. $100 \%$ of doubled image reports happen when subject is seeing the projection of a non-tailored light field (Figure 14(b)). $92 \%$ of cases appeared to be corrected, from the user's perspective, when seen tailored projections ( $\sigma=17 \%$ among 5 rounds of tests with the same subject).

\section{Multi-Focus and Stereoscopic Add-ons}

Multi-focus abilities for 3D scenes require converging light rays from out-of-focus objects to points in front of and behind the retina (Figure 15). Tailored light fields for single-depth planar projections are computed using the subject's eye aberrations and a picture of the expected view only. To create a multi-focus tailored display, a depth component for each retinal point $R$ must be added to the axial length the eye $(a)$ on the Equation 1. An additional depth map $I_{d p t}(R)$ must be provided as input.

Since the tailoring process changes scene depths to the subject's focal range, the scene depth $I_{d p t}(R)$ representation must be independent of global coordinates. We propose to represent relative distances among object depths in diopters. According to our experience, the refocusing feeling of $1 D$ is the same for several distances between two objects. Accommodation change from an object at optical infinity $(0 D)$ to one at $1 \mathrm{~m}(1 D)$ creates the same focusing feeling than objects from $33 \mathrm{~cm}(3 D)$ to $25 \mathrm{~cm}(4 D)$. Also, accommodation speed is measured in diopters per second, which dissociates velocity from a global coordinate [Charman and Heron 2000]. $I_{d p t}(R)$ in diopters preserves our affine transformation. Thus, $a$ in Equation 1 changes to $1 /\left(1 / a+I_{d p t}(R)\right)$, where $I_{d p t}$ is the scenedepth map. Equation 7 supports the scene depth as:

$$
\begin{aligned}
{\left[\begin{array}{cccc}
S_{2_{1}} k_{1} & \cdots & S_{2_{r}} k_{1} \\
S_{2_{1}} k_{2} & \cdots & S_{2_{r}} k_{2} \\
\vdots & \ddots & \vdots \\
S_{2_{1}} k_{n} & \cdots & S_{2_{r}} k_{n}
\end{array}\right]=\left[\begin{array}{cccc}
\frac{-t}{a} & t k_{1} & -t & B_{k_{1}} \\
\frac{--t}{a} & t k_{2} & -t & B_{k_{2}} \\
\vdots & \vdots & \vdots & \vdots \\
\frac{-t}{a} & t k_{n} & -t & B_{k_{n}}
\end{array}\right]\left[\begin{array}{ccc}
R_{1} & \cdots & R_{r} \\
I_{d p t}\left(R_{1}\right) & \cdots & I_{d p t}\left(R_{r}\right) \\
R_{1} I_{d p t}\left(R_{1}\right) & \cdots & R_{r} I_{d p t}\left(R_{r}\right) \\
1 & \cdots & 1 \\
1 &
\end{array}\right] } \\
B_{k}=\frac{t k}{a}-\frac{t k}{f(k)}+k,
\end{aligned}
$$

where positive values on $I_{d p t}$ bring objects closer to the viewer. All units are in meters. Figure 15 also shows two examples of tailoring with multi-focus. On the bottom row, one can create a calibration trick to known where subjects are focusing: show numbers at many depths and ask the subject which number (s)he sees.

Stereoscopy can be achieved by shooting two warped images per LCD pin-hole. For the sake of simplicity, our derivation up to now assumed that the eye is centered with the light-field display (optical axis). For stereoscopy, the system will be aligned with the nose. Equation 1 must be adjusted accordingly.

\section{Conclusion and Discussion}

We propose novel multi-depth displays that compensate for vision aberrations and improve visual acuity without the need of glasses or contact lenses. It supports spatial (higher-order aberrations and cataracts) and time-varying (e.g., daily changes on refractive error from diabetes) optical corrections with no moving parts using offthe-shelf components, in real time. To the best of our knowledge, this is the first display technology capable of adjusting itself to a given subject's visual conditions. Tailored displays decompose virtual objects in many focal depths according to wavefront aberrations of sectors of the eye's aperture. As a single point of focus can be distributed into many depths, it creates multi-focus multi-depth displays. Validation with modified LCD-stacked or lenticular-based light-field displays are performed. Our user experiments attest the

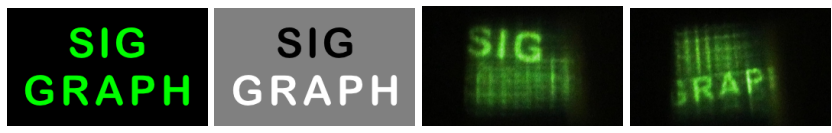

(a) Retinal Image (b) Depth Map

(c) Simul. -0.5D (d) Simul. +0.5D
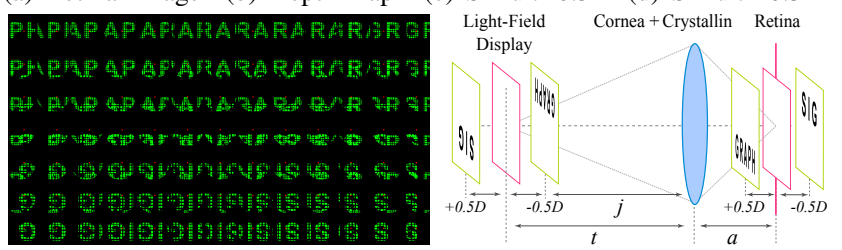

(e) $L C D_{1}$ (green) - $L C D_{2}$ (red)
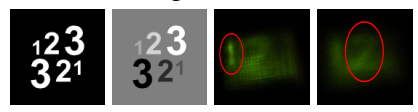

(f) Tailoring Optical Diagram

(g) Inp (h) Dpt (i) -0.5

(j) -1
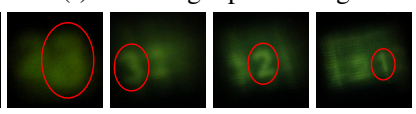

Figure 15: Multi-focus tailored light field. The retinal image (a) and the depth map $(b)$ are inputs to the method. White pixels in $(b)$ represent $-0.5 D$ and black to $+0.5 D$. (e) shows the $L C D_{1}$ and $\mathrm{LCD}_{2}$ images for the projector prototype as green and red colors. Both depths are rendered on top of each other. $(c)$ and $(d)$ simulates an individual focusing $0.5 \mathrm{D}$ in front and behind the display. The display was placed at $t=42 \mathrm{~cm}$ from the eye. Virtual image of $(d)$ is created in front of the display $(j=35 \mathrm{~cm})$ while $(c)$ behind it $(j=53 \mathrm{~cm})$, inverting SIG on Figure $(e)$. The bottom row shows a multi-focus correction for figure $(g)$ with 6 levels $((i)-(n))$ on the depth map ( $h)$ : from $1.1 \mathrm{~m}(k)$ to $25 \mathrm{~cm}$ (l) from the eye $(t=42 \mathrm{~cm})$.

system's ability to create corrected versions of virtual objects to target given eye aberrations. We have shown that currently-available high-resolution displays can create colored virtual objects at a resolution around 1-arc minute, which is the human eye's standard acuity. We validate our method with small binary images and bigger colorful scenes in several environments: (i) presbyopic, where the subject cannot focus close (Figure 8); (ii) myopic, where the subject cannot focus far (Figures 7 and 12); (iii) astigmatic, where subject cannot sharply focus (Figure 11), and (iv) cataracts (Figure 9). User evaluations with spherical (Figure 13(a)), astigmatism (Figure 13(b)) and high-order aberrations (Figure 14) reveal users' preference for our technique.

Limitations Just like glasses-free 3D displays, our approach requires the subject's eyes (and thus head) to be fixed relative to the tailored display. Nevertheless, we anticipate real-time eye-tracking technology would add significant observer movement flexibility. As with other similar designs, our dual stacked LCD has some limitations with regards to brightness, contrast, diffraction, and spatial vs. angular resolution tradeoffs, which are directly affected by the technology used [Dodgson 2009]. The virtual image resolution is a function of the display's angular resolution and the distance from the display to the eye. Tailored displays require high-resolution pixel panels in terms of pixel-per-inch. Retinal and other ocular diseases may affect our results. Changes in pupil size may distort our brightness equalization. However, these variations are easily trackable with a single camera or predicted with models for pupil light reflex [Pamplona et al. 2009]. We believe that the lack of total sharpness in our results is due to: (i) lack of precision in the assembly of our hand-made prototypes, and (ii) the usage of a very tiny portion of the camera's sensor. Most of our results are cropped images that used only $0.55 \%$ of the area of the camera's sensor. Lastly, we presume relatively long viewing distances so that we can assume: (i) a locally flat cornea, (ii) a flat retina or, similarly, a curved focal plane, (iii) isotropic distribution of light among cast rays, and (iv) an eye modeled as a calibrated thin lens system. 
Applications We believe the evolution of this technology will lead to new tailorable user experiences. Our work is inspired by consumer light-field cameras that have realized that exceeding sensor resolution is redundant and can instead be exploited for refocusing. We realize that current trend in display resolution will also create redundant angular resolution for human consumption and it can instead be used to provide new functionality (e.g., vision corrected displays). There are several immediate usages for our work on today's products that do not require significant technological advances. These include head-mounted displays, digital wrist devices, tracking and monitoring tools, small-screen cell phones, and music players. All these can be efficiently built with the inexpensive LCD panels used in our prototypes. We propose its usage in all daily tasks where eyewear is not convenient. Checking the time or speed while running is hard for farsighted individuals, who do not carry reading glasses during these activities. When applied to phones and wrist watches, tailored displays could remove the need to carry reading glasses and the annoying act of putting them on and off just to check why the phone is beeping. Head-mounted displays could provide improved vision and create multi-focus environments inside the subject's accommodation range without moving parts. Tailoring displays have potential to affect several activities where a multi-focus function is required but undoable, such as in tailoring the dashboard of the car to avoid reading glasses for farsighted individuals. Finally, for people with high-order aberrations or cataracts (which cannot be corrected for with eyeglasses), tailoring displays are an interesting alternative to surgeries (which may not be recommended due to patients heart conditions, etc).

Tailored monitors would require close-to gigapixel displays (23" on 1,857-PPI requires 800-megapixel LCDs) and thus several improvements in current hardware and software. Although the LCD panel industry is not evolving as fast as its digital camera sensor counterpart, we hope that by introducing new uses for ultra-highresolution giga-pixel displays we show that there is a clear market to be explored. Since the ever-increasing market value for highquality $3 \mathrm{D}$ displays and HD-ready mobile phones is expected to make higher-resolution LCDs ubiquitous, tailoring methods are expected to grow as more technology becomes available.

Future Works We hope the proposed method contributes for future multi-focal techniques that avoid the mismatch between convergence and accommodation on parallax barriers. Tailored displays could diminish eye strain problems of current displays. Timemultiplexed displays may be used to eliminate loss of spatial resolution [Lanman et al. 2010]. Other methods of optimizing $L C D_{1}$ and $L C D_{2}$ for brightness such as matrix factorization are left for future work. Equation 3 could also account for the Stiles-Crawford effect and for anisotropic light boxes that may optimize perception. Other conditions, such as color vision deficiencies [Machado et al. 2009] (recoloring), retinal displacement and detachment (sensor warping) could be included in the tailoring pipeline. Eye tracking systems and physiologically based models for the eye [Pamplona et al. 2009] can provide real-time data, such as the distance eyedisplay, eye angle and pupil size for the best experience on using our displays. Further user evaluations to assess dynamic contents, a quantitative error analysis of diffraction (beyond geometric optics), and the 3D perception/accommodation of stereo/multi-focus prototypes are required. Additional research is required for applying tailored displays in multi-user interactive environments (e.g., several eyes looking at a public tailored display) and the use of multiple points of view to maximize the search for the best viewing angle. We hope the future of glasses-free 3D displays also accounts for user's eyeglasses. Color calibration for the wavelength profiles of red, green and blue channels would enhance the image being displayed. New technologies to display light-fields, such as an array of nano antennas [Yu et al. 2011], may lead to new trends on our field. Interactive measuring techniques for refractive error [Pamplona et al. 2010] can be improved by applying tailoring to distort their displayed patterns on the fly. A wavelength-dependent tailoring process could create new insights on the eye accommodation behavior for natural scenes when refractive variations among wavelengths are close to null. Convergence-based 3D displays with multi-focus and tailoring features can lead to a new ultra-resolution vision-enhanced 3D technology.

Can tailored displays replace eyeglasses? Eyeglasses provide better vision quality than our current prototypes (and people need them to look around their environments, not only at displays). This paper provides an acuity enhancement option to avoid the need for corrective glasses in activities with (predominantly) relatively short durations. Although we state and validated that images are enhanced for a particular subject, that does not mean that one should avoid optical corrections at all times. Some lenses, like hard contacts for keratoconus, are prescribed to help treat a given visual condition. Studies on how our display could help ocular treatments instead of the current practice are left for future research.

The ultimate tailored display would equalize a designed user experience among an audience. Each individual intakes and interprets sensory stimuli in slightly different ways. The computer's ability to enhance an individual's focusing dexterity could also be applied to hearing, taste, smell, touch, temperature, and time. The individually-enhanced stimuli would compensate for variations in one's ability to accurate sense them.

Acknowledgments Vitor and Manuel acknowledge CNPqBrazil fellowships 142563/2008-0, 308936/2010-8, 480485/20100 , and 308936/2010-8. Daniel is supported by NSF CNS 0913875 and Ramesh by Alfred P Sloan Foundation fellowship, DARPA Young Faculty Award and Media Lab consortium sponsors. iPad photo by Di Bédard, and video voiceover by Cody Sumter.

\section{References}

Akeley, K., Watt, S. J., Girshick, A. R., And Banks, M. S. 2004. A stereo display prototype with multiple focal distances. In SIGGRAPH 2004, I804-813.

Alonso Jr., M., Barreto, A., And Adjouadi, M. 2007. Development and evaluation of a custom display compensation method for computer users based on individual visual characteristics. In 16th Int. Conf. Comp.

BARSKY, B. A. 2004. Vision-realistic rendering. In $A P G V, 73-81$.

CAmp, J., Maguire, L., And RobB, R. 1990. An efficient ray tracing algorithm for modeling visual performance from corneal topography. In Proc. Vis. in Bio. Comp., 278-285.

CAMPBELL, C. 2010. Relative importance of sources of chromatic refractive error in the human eye. J. Opt. Soc. Am. A 27(4).

Charman, W., and Heron, G. 2000. On the linearity of accommodation dynamics. Vision Research 40(15), 2057-2066.

Damera-Venkata, N., And Chang, N. L. 2009. Display supersampling. ACM TOG 28, 9:1-19.

DEERING, M. F. 2005. A photon accurate model of the human eye. In SIGGRAPH 2005, vol. 24(3), 649-658.

Didyk, P., Eisemann, E., Ritschel, T., Myszkowski, K., AND SEIDEL, H.-P. 2010. Apparent display resolution enhancement for moving images. In SIGGRAPH 2010, 113:1-8.

Dodgson, N. 2009. Analysis of the viewing zone of multi-view autostereoscopic displays. In SPIE SD\&A, vol. 4660, 254-265. 
Donnelly, W., Pesudovs, K., Marsack, J., SArver, E., AND APPlegate, R. 2004. Quantifying scatter in ShackHartmann images to evaluate nuclear cataract. J Refract Surg 20(5), S515-S522.

Douali, M. G., AND Silver, J. D. 2004. Self-optimised vision correction with adaptive spectacle lenses in developing countries. Ophthal Physiol Opt 24.

EDPRG. 2004. Prevalence of cataract and pseudophakia/aphakia among adults in the united states. Arch Ophthalmol 122.

Goldring, E., Cain, J., Larson, K., Price, L., Smith, L., RAYEJ, S., AND CAVAllerano, J. 2006. Enhanced visual experiences and seeing hardware for reduced vision. Optom. 77(2).

Hoffman, D. M., Girshick, A. R., Akeley, K., And Banks, M. S. 2005. Vergence-accommodation conflicts hinder visual performance and cause visual fatigue. J. Vis. 5, 10, 834-862.

HuAng, F.-C., AND BARSK, B. A. 2011. A framework for aberration compensated displays. Tech. Rep. UCB/EECS-2011-16.

Isaksen, A., McMillan, L., And Gortler, S. J. 2000. Dynamically reparameterized light fields. In SIGGRAPH 2000.

IsONO, H., YASUDA, M., AND SASAZAWA, H. 1993. Autostereoscopic 3-D display using LCD-generated parallax barrier. Electr. Comm. Japan 76(7), 77-84.

IVES, F. E., 1903. Parallax stereogram \& process of making same. US Patent 725567.

JaYnes, C., AND RAmakrishnan, D. 2003. Super-resolution composition in multi-projector displays. In IEEE ProCams.

JeOnG, T. M., Ko, D.-K., AND LEE, J. 2005. Generalized raytransfer matrix for an optical element having an arbitrary wavefront aberration. Opt. Lett. 30(22), 3009-11.

Lanman, D., Hirsch, M., Kim, Y., AND RASKAR, R. 2010. Content-adaptive parallax barriers. ACM TOG 29(6), 163:1-10.

Levoy, M., Chen, B., Vaish, V., Horowitz, M., MCDOWALL, I., AND BOLAS, M. 2004. Synthetic aperture confocal imaging. ACM TOG 23, 825-834.

Levoy, M., Zhang, Z., AND McDowall, I. 2009. Recording and controlling the 4D light field in a microscope using microlens arrays. J Microsc 235(2), 144-162.

Liang, J., Grimm, B., Goelz, S., AND Bille, J. 1994. Objective measurement of wave aberrations of the human eye with a Hartmann-Shack sensor. JOSA A 11(7), 1949-1957.

Liang, J., Williams, D. R., AND Miller, D. T. 1997. Supernormal vision \& high-resolution retinal imaging through adaptive optics. JOSA A 14(11), 2884-2892.

LiPPMANN, G. 1908. épreuves réversibles donnant la sensation du relief. $J$ Phys 7, 821-825.

LiU, S., AND HuA, H. 2009. Time-multiplexed dual-focal plane head-mounted display with a liquid lens. Opt. Lett. 34(11), 1642.

Machado, G. M., Oliveira, M. M., And Fernandes, L. A. F. 2009. A physiologically-based model for simulation of color vision deficiency. IEEE Trans. Vis. Comp. Graph. 15(6).

MADDOX, E. E. 1886. Investigations in the relation between convergence and accommodation of the eyes. J Anat Physiol 20(3).

Ming Dai, G., CAmpbell, C. E., Chen, L., ZhaO, H., AND ChernyaK, D. 2009. Wavefront propagation from one plane to another with the use of zernike polynomials and taylor monomials. Appl Opt 48(3), 477-488.

Morgan, I. G., Ohno-Matsui, K., And SAw, S.-M. 2012. Myopia. The Lancet 379: 1739-48.

NG, R., AND Hanrahan, P. 2006. Digital correction of lens aberrations in light field photography. In SPIE IODC, vol. 6342.

NG, R., Levoy, M., Brédif, M., Duval, G., Horowitz, M., AND HANRAHAN, P. 2005. Light field photography with a hand-held plenoptic camera. Tech. Rep. CTSR 2005-02.

NG, R. 2005. Fourier slice photography. ACM TOG 24, 735-744.

Pamplona, V. F., Oliveira, M. M., And Baranoski, G. 2009. Photorealistic models for pupil light reflex and iridal pattern deformation. ACM TOG 28(4), 106.

Pamplona, V. F., Mohan, A., Oliveira, M. M., And RASKAR, R. 2010. NETRA: interactive display for estimating refractive errors and focal range. In SIGGRAPH 2010, 77:1-8.

Pamplona, V. F., Passos, E. B., ZizKa, J., Oliveira, M. M., LaWson, E., Clua, E., AND Raskar, R. 2011. CATRA: interactive measuring and modeling of cataracts. In SIGGRAPH 2011, 47:1-8.

PorterfIELD, W. 1759. A Treatise on the Eye: the manner \& phenomena of vision.

Rolland, J. P., Krueger, M. W., And Goon, A. 2000. Multifocal planes head-mounted displays. Appl Opt 39(19), 3209-15.

Rozema, J. J., Dyck, D. E. V., AND TAssignon, M.-J. 2005. Clinical comparison of 6 aberrometers. part 1: Technical specifications. JCRS 31, 6, $1114-1127$.

Rucker, F. J., And Kruger, P. B. 2006. Cone contributions to signals for accommodation and the relationship to refractive error. Vision Research 46, 3079-3089.

SCHAEFFEL, F. 2006. Myopia: The importance of seeing fine detail. Curr. Biol. 16(7), R257-R259.

Schwiegerling, J. 2000. Theoretical limits to visual performance. Surv Ophthalmol 45(2), 139-146.

SCHWIEGERLING, J. 2004. Field guide to visual and ophthalmic optics. SPIE.

SugiURA, N., AND Morita, S. 1993. Variable-focus liquid-filled optical lens. Appl Opt. 32(22).

Thibos, L., Qi, X., And Miller, D. T. 1999. Vision through a liquid-crystal spatial light modulator. In Adaptive Optics for Industry and Medicine.

Vaish, V., Wilburn, B., Joshi, N., AND Levoy, M. 2004. Using plane + parallax for calibrating dense camera arrays. In IEEE CVPR, 2-9.

Wetzstein, G., LANMAn, D., Heidrich, W., And Raskar, R. 2011. Layered 3D: Tomographic image synthesis for attenuation-based light field and high dynamic range displays. ACM TOG 30, 4 .

WHO, 2005. State of the world's sight. vision 2020: the right to sight 1999-2005.

Yu, N., Genevet, P., Kats, M. A., Aieta, F., Tetienne, J.P., CAPAsso, F., AND GaburRo, Z. 2011. Light propagation with phase discontinuities. Science 334 (6054), 333-337. 